\title{
THE WEIERSTRASS CONDITION FOR MULTIPLE INTEGRAL VARIATIONAL PROBLEMS INVOLVING HIGHER DERIVATIVES ${ }^{1}$
}

LAWRENCE M. GRAVES

1. Introduction. We consider the problem of minimizing a multiple integral

$$
I=\int_{G} f(x, z, D z) d x=\int \cdots \int f(x, z, D z) d x_{1} \cdots d x_{n},
$$

where $x=\left(x_{1}, \cdots, x_{n}\right), z=\left(z_{1}, \cdots, z_{q}\right), z$ is a function of $x$, and $D z_{k}$ denotes the various partial derivatives of $z_{k}$ with respect to the $x_{j}$ up to order $\nu_{k}$. When it is necessary to be more explicit, we shall let $i$ denote an $n$-dimensional vector with nonnegative integer coordinates, and write

$$
D^{i}=\prod_{j=1}^{n} D_{x_{j}}^{i_{j}}
$$

We set $|i|=\sum_{j} i_{j}$, and if $|i|=\nu_{k}$, denote $D^{i} \boldsymbol{z}_{k}$ by $p_{\mathbf{k}}^{i}$. These are the derivatives of $z_{k}$ of the highest order that appear.

It is supposed that each $z_{k}$ and its derivatives up to order $\nu_{k}-1$ are continuous on a fixed domain $G$ and take prescribed boundary values on the boundary $G^{*}$ of $G$, and that the derivatives of $z_{k}$ of order $\nu_{k}$ are piecewise continuous. We assume that the integrand $f$ is continuous and has continuous partial derivatives with respect to the arguments $p_{k}^{i}$, for points $(x, z, D z)$ interior to a domain $T$. In the Weierstrass $\mathcal{E}$-function, only the arguments $p_{\boldsymbol{k}}^{i}$ are varied. Hence we shall define $D z+P$ by the formula

$$
\begin{aligned}
(D z+P)_{k}^{i} & =D^{i} z_{k} \quad \text { for }|i|<\nu_{k} \\
& =D^{i} z_{k}+P_{k}^{i} \quad \text { for }|i|=\nu_{k},
\end{aligned}
$$

and assume for simplicity that the domain $T$ is such that $(x, z, D z+P)$ is in $T$ whenever $(x, z, D z)$ is in $T$. Then we define

$\mathcal{E}(x, z, D z, D z+P)=f(x, z, D z+P)-f(x, z, D z)-\sum_{i, k} P_{k}^{i} f_{p_{k} i}^{i}(x, z, D z)$,

Received by the editors November 23, 1959.

1 This work was supported by the Office of Ordnance Research under Contract No. DA-11-022-ORD-1833. 
where the summation index $k$ runs from 1 to $q$, and $i$ varies over the set $|i|=\nu_{k}$.

We shall show that if $I$ is a minimum then

$$
\mathcal{E}(x, z, D z, D z+P) \geqq 0
$$

whenever $P$ has the form

$$
P_{k}^{i}=C_{k} \prod_{j=1}^{n}\left(\alpha_{j}\right)^{i j}
$$

where $C_{k}$ and $\alpha_{j}$ are arbitrary.

We may restrict attention to a point $x$ of $G$ near which all derivatives of $z$ which appear are continuous, and consider only variations $\zeta$ of $z$ which vanish outside a neighborhood of $x$. Then if we put

$$
\begin{aligned}
\bar{f}(x, \zeta, D \zeta) & =f(x, z+\zeta, D z+D \zeta)-f(x, z, D z), \\
\bar{I}(\zeta) & =\int \bar{f} d x,
\end{aligned}
$$

we see that in $(x, \zeta)$-space, the minimizing manifold is $\zeta=0$, and $\bar{f}(x, 0,0)=0$. By a translation we may also suppose that the point $x$ under consideration is the origin. We replace $\zeta$ by $z$ and $\bar{f}$ by $f$, and understand in the proofs that any argument of $f$ or its partial derivatives which is not written is zero. In $\$ 2$ we give the proof for the case $q=2, \nu_{1}=1, \nu_{2}=2$, and in $\S 3$ treat the general case. The method of proof is an extension of that given by the author for the case when only first derivatives appear. ${ }^{2}$

2. A special case. We consider here an integrand

$$
f\left(x, z_{1}, z_{2}, D z_{1}, D z_{2}, D^{2} z_{2}\right) \text {, }
$$

where $D^{2} z_{2}$ stands for all the second derivatives $D_{x_{j}} D_{x_{m}} z_{2}$, and no derivatives appear which are of higher order than those indicated. Let

$$
L_{0}=\sum_{j=1}^{n} \alpha_{j} x_{j},
$$

where for convenience $\alpha$ is chosen as a unit vector, and for a small $b>0$ and $|x| \leqq b$ let

$$
\phi=\left[1+L_{0}^{2}-|x|^{2}\right]^{1 / 2}-\left[1-b^{2}\right]^{1 / 2},
$$

where $|x|$ denotes the Euclidean length of the vector $x$. (Note that

2 See Duke Math. J. vol. 5 (1939) pp. 656-660. 
$|i|$ was defined differently.) Then $\phi$ has bounded partial derivatives of all orders, and $\phi$ and its first partial derivatives approach zero uniformly with $b$. Let $1=\epsilon_{0}>\epsilon_{1}>\epsilon_{2}>\epsilon_{3}=0$, and set

$$
\begin{aligned}
& L_{1}=L_{0}+\left(\epsilon_{1}-1\right) \phi, \\
& L_{2}=L_{0}+\left(\epsilon_{2}-1\right) \phi, \\
& L_{3}=L_{0}-\phi .
\end{aligned}
$$

Then the loci $L_{\beta}=0,(\beta=0,1,2,3)$, bound three adjacent domains $R_{0}, R_{1}, R_{2}$ in $x$-space, defined by

$$
R_{\beta}=\left[x \mid L_{\beta+1}<0<L_{\beta}\right] .
$$

If $V=V(b)$ denotes the volume of $R=R_{0}+R_{1}+R_{2}$, then the volume of $R_{\beta}$ is $V_{\beta}=\left(\epsilon_{\beta}-\epsilon_{\beta+1}\right) V$, as is readily verified by considering the special case $\alpha_{1}=1, \alpha_{j}=0$ for $j>1$. Also $V$ tends to zero with $b$.

The variations of the minimizing manifold $z=0$ are constructed as follows. Let $A_{10}, A_{20}$ be arbitrary constants, and let $A_{11}, A_{21}, A_{22}$ denote functions of $\epsilon_{1}, \epsilon_{2}$, to be determined. Set

$$
\begin{array}{rlrl}
z_{1} & =A_{10} L_{0} & & \text { on } R_{0}, \\
& =A_{10} L_{0}+A_{11} L_{1} & & \text { on } R_{1}+R_{2}, \\
z_{2} & =A_{20} L_{0}^{2} & & \text { on } R_{0}, \\
& =A_{20} L_{0}^{2}+A_{21} L_{1}^{2} & & \text { on } R_{1}, \\
& =A_{20} L_{0}^{2}+A_{21} L_{1}^{2}+A_{22}^{2} L_{2}^{2} \quad \text { on } R_{2}, \\
z_{1} & =z_{2}=0 \text { outside } R .
\end{array}
$$

Then $z_{1}$ is continuous except possibly along $L_{3}=0$, and $z_{2}$ and its first partial derivatives are continuous except possibly along $L_{3}=0$. Sufficient conditions for the required continuity along $L_{3}=0$ are

$$
\begin{aligned}
A_{10}+\epsilon_{1} A_{11} & =0, \\
A_{20}+\epsilon_{1}^{2} A_{21}+\epsilon_{2} A_{22} & =0, \\
A_{20}+\epsilon_{1} A_{21}+\epsilon_{2} A_{22} & =0,
\end{aligned}
$$

since $L_{0}=\phi, L_{1}=\epsilon_{1} \phi, L_{2}=\epsilon_{2} \phi$ on $L_{3}=0$.

Now when $b$ tends to zero, so do $\phi$, each $L_{\beta}, z_{1}, z_{2}$, and each $D_{x_{j}} \phi$, and hence

$$
\begin{aligned}
& D_{x_{j}} L_{\beta} \rightarrow D_{x_{j}} L_{0}=\alpha_{j}, \\
& D_{x_{j}} L_{\beta}^{2} \rightarrow 0,
\end{aligned}
$$




$$
\begin{aligned}
D_{x_{j}} D_{x_{m}} L_{\beta}^{2} & \rightarrow 2 \alpha_{j} \alpha_{m}, \\
D_{x_{j}} z_{1} & \rightarrow A_{10} \alpha_{j} \quad \text { on } R_{0}, \\
& \rightarrow\left(A_{10}+A_{11}\right) \alpha_{j} \quad \text { on } R_{1}+R_{2}, \\
D_{x_{j}} z_{2} & \rightarrow 0 \\
D_{x_{j}} D_{x_{m}} z_{2} & \rightarrow 2 A_{20} \alpha_{j} \alpha_{m} \quad \text { on } R_{0}, \\
& \rightarrow 2\left(A_{20}+A_{21}\right) \alpha_{j} \alpha_{m} \quad \text { on } R_{1}, \\
& \rightarrow 2\left(A_{20}+A_{21}+A_{22}\right) \alpha_{j} \alpha_{m} \quad \text { on } R_{2} .
\end{aligned}
$$

Since

$$
\frac{I}{V}=\frac{1-\epsilon_{1}}{V_{0}} \int_{R_{0}} f d x+\frac{\epsilon_{1}-\epsilon_{2}}{V_{1}} \int_{R_{1}} f d x+\frac{\epsilon_{2}}{V_{2}} \int_{R_{2}} f d x,
$$

we find from $I(0)=0=$ minimum of $I(b)$ that

$$
\begin{aligned}
0 \leqq & \left(1-\epsilon_{1}\right) f\left(A_{10} \alpha_{j}, 2 A_{20} \alpha_{j} \alpha_{m}\right) \\
& +\left(\epsilon_{1}-\epsilon_{2}\right) f\left[\left(A_{10}+A_{11}\right) \alpha_{j}, 2\left(A_{20}+A_{21}\right) \alpha_{j} \alpha_{m}\right] \\
& +\epsilon_{2} f\left[\left(A_{10}+A_{11}\right) \alpha_{j}, 2\left(A_{20}+A_{21}+A_{22}\right) \alpha_{j} \alpha_{m}\right] .^{3}
\end{aligned}
$$

This inequality may be regarded as a generalized form of the Weierstrass condition, in which no partial derivatives of the integrand $f$ appear. We obtain the ordinary form of the condition by dividing by $\left(1-\epsilon_{1}\right)$ and letting $\epsilon_{1}$ tend to one. In order to evaluate this limit we need the derivatives $A_{11}^{\prime}, A_{21}^{\prime}$ and $A_{22}^{\prime}$ of $A_{11}, A_{21}$ and $A_{22}$ with respect to $\epsilon_{1}$ at $\epsilon_{1}=1$. Let $M_{\beta}$ be the cofactor of $s_{\beta}$ in the determinant

$$
\left|\begin{array}{ccc}
s_{0} & s_{1} & s_{2} \\
1 & \epsilon_{1} & \epsilon_{2} \\
1 & \epsilon_{1}^{2} & \epsilon_{2}
\end{array}\right| .
$$

Then from the equations (3),

$$
A_{21}=A_{20} M_{1} / M_{0}, \quad A_{22}=A_{20} M_{2} / M_{0} .
$$

Also at $\epsilon_{1}=1, M_{1}=-M_{0}, M_{2}=0$, and

$$
\frac{\partial}{\partial \epsilon_{1}} \frac{M_{1}}{M_{0}}=\frac{M_{0}^{\prime}}{M_{0}}, \quad \frac{\partial}{\partial \epsilon_{1}} \frac{M_{2}}{M_{0}}=\frac{M_{2}^{\prime}}{M_{0}},
$$

$$
\begin{array}{lll}
A_{21}^{\prime}=A_{20} M_{0}^{\prime} / M_{0}, & A_{22}^{\prime}=A_{20} M_{2}^{\prime} / M_{0}, & A_{11}^{\prime}=A_{10}, \\
A_{10}+A_{11}=0 & A_{20}+A_{21}=0, & A_{20}+A_{21}+A_{22}=0 .
\end{array}
$$

' Here the arguments $x, z_{1}, z_{2}, D z_{2}$ of $f$, which are all zero, have been omitted. 
In this way we obtain from (4) the inequality

$$
\begin{aligned}
0 \leqq & f\left(A_{10} \alpha_{j}, 2 A_{20} \alpha_{j} \alpha_{m}\right) \\
& +\left(\epsilon_{2}-1\right)\left[\sum_{j} f_{p_{1 j}} A_{10} \alpha_{j}+\sum_{j, m} f_{p_{2 j m}} 2 A_{20} M_{0}^{\prime} \alpha_{j} \alpha_{m} / M_{0}\right] \\
& -\epsilon_{2}\left[\sum_{j} f_{p_{1 j}} A_{10} \alpha_{j}+\sum_{j, m} f_{p_{2 j m}} 2 A_{20}\left(M_{0}^{\prime}+M_{2}^{\prime}\right) \alpha_{j} \alpha_{m} / M_{0}\right] .
\end{aligned}
$$

Now at $\epsilon_{1}=1,\left(1-\epsilon_{2}\right) M_{0}^{\prime}+\epsilon_{2}\left(M_{0}^{\prime}+M_{2}^{\prime}\right)=M_{0}$, so this becomes

$$
0 \leqq f\left(A_{10} \alpha_{j}, 2 A_{20} \alpha_{j} \alpha_{m}\right)-\sum_{j} f_{p_{1 j}} A_{10} \alpha_{j}-\sum_{j, m} f_{p_{2 j m}} 2 A_{20} \alpha_{j} \alpha_{m}
$$

or

$$
0 \leqq f\left(p_{1}, p_{2}\right)-\sum_{j} p_{1 j} f_{p_{1 j}}-\sum_{j, m} p_{2 j m} f_{p_{2 j m}},
$$

where $p_{1 j}=A_{10} \alpha_{j}, p_{2 j m}=2 A_{20} \alpha_{j} \alpha_{m}$, and the arguments of the partial derivatives of $f$ are those along the minimizing manifold $z_{1}=z_{2}=0$.

3. The general case. We let $\mu$ denote the maximum $\nu_{k}$, and select $\epsilon_{\beta}$ satisfying

$$
1=\epsilon_{0}>\epsilon_{1}>\cdots>\epsilon_{\mu}>\epsilon_{\mu+1}=0 .
$$

With $L_{0}$ and $\phi$ chosen as in $\S 2$, we set

$$
L_{\beta}=L_{0}+\left(\epsilon_{\beta}-1\right) \phi .
$$

There are now $\mu+1$ domains $R_{\beta}$ defined by (2), and the domain $R$ which is their union is defined by the inequality $0<L_{0}<\phi$. On $R_{\beta}$ we set

$$
z_{k}=\sum_{\sigma=0}^{\lambda} A_{k \sigma} L_{\sigma}^{\nu_{k}}
$$

where $\lambda$ is the lesser of $\beta$ and $\nu_{k}, A_{k 0}$ is arbitrary, and the remaining $A_{k \sigma}$ are to be determined as multiples of $A_{k 0}$. Outside of $R$ we set $z_{k}=0$. The functions $z_{k}$ and their partial derivatives up to order $\nu_{k}-1$ are obviously continuous along the manifolds $L_{\beta}=0$ for $\beta=0,1, \cdots, \mu$. To assure the required continuity along the manifold $L_{\mu+1}=0$, it is sufficient to require that the $A_{k \sigma}$ satisfy the equations

$$
\sum_{\sigma=0}^{\nu_{k}} A_{k \sigma \epsilon_{\sigma}}^{p}=0, \quad \rho=1, \cdots, \nu_{k},
$$


as may be verified by observing that when $L_{\mu+1}=0, L_{\sigma}=\epsilon_{\sigma} \phi, D L_{0}=\alpha$, $D L_{\sigma}=D L_{0}+\left(\epsilon_{\sigma}-1\right) D \phi, D^{2} L_{\sigma}=\left(\epsilon_{\sigma}-1\right) D^{2} \phi$, etc. Hence we take

$$
A_{k \sigma}=A_{k 0} M_{k \sigma} / M_{k 0} \text {, }
$$

where $M_{k \sigma}$ is the cofactor of $s_{o}$ in the determinant

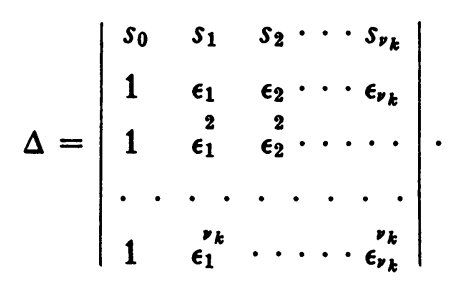

When $b$ tends to zero, each $z_{k}$, with its derivatives up to order $\nu_{k}-1$, tends to zero, and for $|i|=\nu_{k}, D^{i} z_{k}$ tends to $p_{\mathbf{k} \beta}^{i}$ on $R_{\beta}$, where

(5) $\quad p_{k \beta}^{i}=\nu_{k} ! \prod_{j=1}^{n}\left(\alpha_{j}\right)^{i j} \sum_{\sigma=0}^{\lambda} A_{k \sigma}=B_{k}{ }^{i} \sum_{\sigma=0}^{\lambda} A_{k \sigma}=B_{k}{ }^{i} A_{k 0} \sum_{\sigma=0}^{\lambda} M_{k \sigma} / M_{k 0}$.

As in $\S 2$ we may write

$$
\frac{I}{V}=\sum_{\beta=0}^{\mu} \frac{\epsilon_{\beta}-\epsilon_{\beta+1}}{V_{\beta}} \int_{R_{\beta}} f d x
$$

and derive as before the inequality

$$
0 \leqq \sum_{\beta=0}^{\mu}\left(\epsilon_{\beta}-\epsilon_{\beta+1}\right) f\left(p_{\beta}\right) \text {. }
$$

Then we divide (6) by $\left(1-\epsilon_{1}\right)$ and let $\epsilon_{1}$ tend to unity. In order to evaluate the result we observe the following relations.

If in the determinant $\Delta$ we put $s_{0}=s_{1}=1, s_{\sigma}=\epsilon_{\sigma}$ for $\sigma>1$, we find by differentiating the expansion of $\Delta$ on the first row that

$$
\frac{\partial \Delta}{\partial \epsilon_{1}}=\frac{\partial M_{k 0}}{\partial \epsilon_{1}}+\sum_{\sigma=2}^{\nu_{k}} \epsilon_{\sigma} \frac{\partial M_{k \sigma}}{\partial \epsilon_{1}} .
$$

By first subtracting the second row from the first and then differentiating, we find

$$
\frac{\partial \Delta}{\partial \epsilon_{1}}=-M_{k 1}
$$

At $\epsilon_{1}=1$, we find

$$
M_{k 1}=-M_{k 0}, \quad M_{k \beta}=0 \text { for } \beta>1,
$$


(10)

$$
\begin{aligned}
\frac{\partial}{\partial \epsilon_{1}} \frac{M_{k 1}}{M_{k 0}} & =\frac{1}{M_{k 0}} \frac{\partial M_{k 0}}{\partial \epsilon_{1}}, \\
\frac{\partial}{\partial \epsilon_{1}} \frac{M_{k \beta}}{M_{k 0}} & =\frac{1}{M_{k 0}} \frac{\partial M_{k \beta}}{\partial \epsilon_{1}} \quad \text { for } \beta>1 . \\
\frac{\partial}{\partial \epsilon_{1}} p_{k \beta}^{i} & =B_{k}^{i} \frac{A_{k 0}}{M_{k 0}} \frac{\partial}{\partial \epsilon_{1}}\left[M_{k 0}+\sum_{\sigma=2}^{\lambda} M_{k \sigma}\right], \\
\sum_{\beta=1}^{\mu}\left(\epsilon_{\beta}-\epsilon_{\beta+1}\right) \frac{\partial}{\partial \epsilon_{1}} & {\left[M_{k 0}+\sum_{\sigma=2}^{\lambda} M_{k \sigma}\right] } \\
= & \frac{\partial}{\partial \epsilon_{1}}\left[M_{k 0}+\sum_{\sigma=2}^{t_{k}} \epsilon_{\sigma} M_{k \sigma}\right]=M_{k 0} .
\end{aligned}
$$

The last equality follows from (7), (8) and (9). So from (6) we have

$$
\begin{aligned}
0 & \leqq f\left(B_{k}^{i} A_{k 0}\right)-\sum_{\beta=1}^{\mu}\left(\epsilon_{\beta}-\epsilon_{\beta+1}\right) \sum_{k, i} f_{p_{k}} \frac{\partial}{\partial \epsilon_{1}} p_{k \beta}^{i} \\
& =f\left(B_{k}^{i} A_{k 0}\right)-\sum_{k, i} B_{k}^{i} A_{k 0} f_{p_{k}}(0)
\end{aligned}
$$

with the help of (10) and (11). Since by (5)

$$
B_{k}^{i}=\nu_{k} ! \prod_{j=1}^{n}\left(\alpha_{j}\right)^{i j}
$$

and since we have assumed $f(0)=0$, the result has the form given in $\S 1$.

The University of Chicago 